\title{
Finite groups with some CAP-subgroups ${ }^{1}$
}

\author{
Luis M. Ezquerro $(*)$ - Xianhua Li $(* *)$ - YANGming Li $(* * *)$
}

ABstract - A subgroup $A$ of a group $G$ is said to be a CAP-subgroup of $G$ if for any chief factor $H / K$ of $G$, there holds $H \cap A=K \cap A$ or $H A=K A$. We investigate the influence of CAP-subgroups on the structure of finite groups. Some recent results are generalized.

Mathematics Subject Classification (2010). 20D10, 20 D15.

KEYworDS. CAP-subgroup, the smallest generator number of a $p$-group, $p$-supersolvable group.

\section{Introduction}

All groups considered in this paper are finite. We use conventional notions and notation, as in Huppert [7]. $G$ always denotes a finite group, $|G|$ is the order of $G, \pi(G)$ denotes the set of all primes dividing $|G|, G_{p}$ is a Sylow $p$-subgroup of $G$ for some $p \in \pi(G)$.

For a subgroup $A$ of $G$, if $H / K$ is a chief factor of $G$, then we will say that:

(1) $A$ covers $H / K$ if $H A=K A$;

(2) $A$ avoids $H / K$ if $H \cap A=K \cap A$;

1 Project supported in part by NSFC(11271085) and NSF of Guangdong (S2011010004447).

(*) Indirizzo dell'A.: Departamento de Matemáticas, Universidad Pública de Navarra, Pamplona, E-31006, Spain.

E-mail: ezquerro@unavarra.es

(**) Indirizzo dell'A.: Dept. of Math., Suzhou University, Suzhou, 215006, China. E-mail:xhli@suda.edu.cn

(***) Indirizzo dell'A.: Dept. of Math., Guangdong University of Education, Guangzhou, 510310, China.

E-mail: liyangming@gdei.edu.cn 
(3) $A$ has the cover and avoidance properties in $G$, in brevity, $A$ is $a$ $C A P$-subgroup of $G$ ([4]), if $A$ either covers or avoids every chief factor of $G$.

Clearly normal subgroups are CAP-subgroups. Examples of CAPsubgroups in the universe of solvable groups are well-known. The most remarkable CAP-subgroups of a solvable group are perhaps the Hall subgroups. By an obvious consequence of the definition of supersolvable group every subgroup of supersolvable group is a CAP-subgroup. In the literature, a lot of people have investigated the influence of the CAPsubgroups of $G$ on the structure of $G$, please see [3], [4], [5], [6], [9], [11], [12], [13], [14], etc. For example, in [3] the first author has gotten the following results: 1 . ([3, Theorem A]) Let $p$ be a prime, $G$ be a $p$-solvable group. Suppose that all maximal subgroups of the Sylow $p$-subgroups of $G$ are CAP-subgroups of $G$, then $G$ is $p$-supersolvable; 2 . ([3, Theorem C]) Suppose that $G$ is a group and for every prime $p$ in $\pi(G)$ and for every Sylow $p$-subgroup $P$ of $G$, every maximal subgroup of $P$ is a CAPsubgroup of $G$. Then $G$ is supersolvable.

In this paper, we extend Ezquerro's the results at least in three aspects: first, removing the hypotheses that $G$ is $p$-solvable in [3, Theorem A]; secondly, reducing the number of restricted maximal subgroups of Sylow subgroups; in third, giving the unified forms of Ezquerro's results.

Suppose that $P$ is a $p$-group for some prime $p$. Let $\mathcal{M}(P)$ be the set of all maximal subgroups of $P$.

DEFINITION ([10]). Let $d_{p}$ be the smallest generator number of a p-group $P$, i.e., $p^{d_{p}}=|P / \Phi(P)|$. We consider the set $\mathcal{M}_{d_{p}}(P)=\left\{P_{1}, \ldots, P_{d_{p}}\right\}$ of all elements of $\mathcal{M}(P)$ such that

$$
\bigcap_{i=1}^{d_{p}} P_{i}=\Phi(P)
$$

We know that

$$
|\mathcal{M}(P)|=\frac{p^{d_{p}}-1}{p-1}, \quad\left|\mathcal{M}_{d_{p}}(P)\right|=d_{p}
$$

and

$$
\lim _{d_{p} \rightarrow \infty} \frac{\frac{p^{d_{p}}-1}{p-1}}{d_{p}}=\infty
$$


so

$$
|\mathcal{M}(P)|>>\left|\mathcal{M}_{d_{p}}(P)\right|
$$

Our main result is as follows.

MAIN Result. Suppose that $G$ is a group and $p$ is a fixed prime number in $\pi(G)$ and $P$ is a Sylow p-subgroup of $G$. Suppose that every member in $\mathcal{M}_{d_{p}}(P)$ is a CAP-subgroup of $G$. Then either $P$ is of order $p$ or $G$ is p-supersolvable.

\section{Preliminaries}

Lemma 2.1. Let $N$ be a normal subgroup of $G$ and $A$ a CAP-subgroup of $G$. Then:

(1) $A N$ is a CAP-subgroup of $G$;

(2) $A N / N$ is a $C A P$-subgroup of $G / N$;

(3) For any chief series (*) of $G, A$ covers or avoids every chief factor of the series (*) and furthermore, the order of $A$ is the product of the orders of the covered chief factors in the series (*).

Proof. (1) is given in [14, §1, Lemma 1.4]; (2) follows from (1); (3) is clear by the definition of CAP-subgroup.

Lemma 2.2 ([7, I, Hauptsatz 17.4]). Suppose that $N$ is an abelian normal subgroup of $G$ and $N \leq M \leq G$ such that $(|N|,[G: M])=1$. If $N$ is complemented in $M$, then $N$ is complemented in $G$.

LeMma 2.3. Let $P$ be a non-cyclic Sylow p-subgroup of $G$ and $p \in \pi(G)$. Suppose that $\Phi(P)_{G}=1$ and $O_{p}(G)>1$ and suppose that every member in $\mathcal{M}_{d_{p}}(P)$ is a $C A P$-subgroup of $G$. Then:

(1) $N_{p}$ is at most of order p for every minimal normal subgroup $N$ of $G$;

(2) every minimal normal subgroup of $G$ contained in $P$ is of order $p$;

(3) $G=O_{p}(G) \times M$, the semi-direct product of $O_{p}(G)$ with a subgroup $M$ of $G$ and $O_{p}(G)$ is a direct product of normal subgroups of $G$ of order $p$.

Proof. (1) Suppose that $N$ is minimal normal in $G$. For any $P_{i} \in \mathcal{M}_{d_{p}}(P)$, we know that either $N \leq P_{i}$ or $N \cap P_{i}=1$. If $N \leq P_{i}$ for all 
$P_{i} \in \mathcal{M}_{d_{p}}(P)$, then

$$
N \leq \bigcap_{i=i}^{d_{p}} P_{i}=\Phi(P),
$$

which is contrary to the hypotheses that $\Phi(P)_{G}=1$. Hence there exists a $P_{i_{0}} \in \mathcal{M}_{d_{p}}(P)$ such that $N \cap P_{i_{0}}=1$. Since $P_{i_{0}}$ is maximal in $P$, we have $N_{p}$ is at most of order $p$.

(2) It is a corollary of (1).

(3) Let $N_{1}$ be a minimal normal subgroup of $G$ contained in $O_{p}(G)$. Then $N_{1}$ is of order $p$ by (2) and $N_{1} \cap \Phi(P)=1$ by the hypotheses that $\Phi(P)_{G}=1$. Hence there exists a maximal subgroup $S_{1}$ of $P$ such that $N_{1} \cap S_{1}=1$. By Lemma 2.2, $N_{1}$ has a complement $K$ in $G$, i.e., $G=N_{1} K$ and $N_{1} \cap K=1$. Then $O_{p}(G)=N_{1}\left(O_{p}(G) \cap K\right)$. It is easy to see that $O_{p}(G) \cap K$ is normal in $G$ and $P \cap K$ is a Sylow $p$-subgroup of $K$. If $O_{p}(G) \cap K=1$, then our theorem holds. So assume that $O_{p}(G) \cap K \neq 1$. Then we can pick a minimal subgroup $N_{2}$ contained in $O_{p}(G) \cap K$. By (1), $N_{2}$ is of order $p$ and there exists a maximal subgroup $S_{2}$ of $P$ such that $N_{2} \cap S_{2}=1$ by the hypothesis that $\Phi(P)_{G}=1$. Then $P=N_{2} S_{2}=S_{2}\left(O_{p}(G) \cap K\right)=S_{2}(P \cap K)$. Since $\left|(P \cap K):\left(S_{2} \cap K\right)\right|=\left|S_{2}(P \cap K): S_{2}\right|=\left|P: S_{2}\right|=p, S_{2} \cap K$ is a complement of $N_{2}$ in $P \cap K$. Therefore $N_{2}$ has a complement $L$ in $K$ by Lemma 2.2. Then $G=N_{1} K=\left(N_{1} \times N_{2}\right) \times L$. Continuing this process, we have finally $G=O_{p}(G) \times M$ and $O_{p}(G)=N_{1} \times N_{2} \times \cdots \times N_{r}$, where $N_{i}$ is a normal subgroup of $G$ of order $p$.

Let $p$ be a prime and $n>1$ a natural number. If $p^{s}$ divides $n$ but $p^{s+1}$ does not divide $n$, we write $(n)_{p}=p^{s}$. Let $t$ be a prime and $b>1$ and let $k$ be a natural number. If $t, b$ and $k$ satisfy that $t$ divides $b^{k}-1$ but $t$ does not divide $b^{i}-1$ for all $i$ with $1 \leq i<k$, then $k$ is called the order of $b$ module $t$ and is denoted by $\exp _{t}(b)$.

LEMma 2.4. Suppose that $H$ is a nonabelian simple group. If the Sylow $r$-subgroups $H_{r}$ of $H$ are of order $r$, where $r$ is a prime, then the out automorphism group Out $(H)$ of $H$ is a $r^{\prime}$-group.

Proof. Suppose that, in the contrary, the order of $O u t(H)$ is divided by $r$. Obviously $r>2$ by [7, IV Satz 2.8]. We will conduct a contradiction by applying the classification of finite simple groups.

If $H$ is a sporadic simple group, then by [2], $|O u t(H)| \mid 2$. If $H$ is a alternating group, then when $H=A_{6},|O u t(H)|=2^{2}$; when $H \neq A_{6}$, $|\operatorname{Out}(H)|=2$. Hence by $r>2$ and $r|| \operatorname{Out}(H) \mid$, we may assume that $H$ is a 
Lie type simple group over $G F(q)$ with $q=p^{f}$. By [2], $|O u t(H)|=d f g$ and so $r \mid d f g$, where the numbers $d, f, g$ are tabulated in [2, Table 5].

Suppose that $r=p$. By the order of Lie type simple groups and $\left|H_{r}\right|=r$, we have $H=A_{1}(p)$. But when $H=A_{1}(p), \quad|O u t(H)|=2$, $r \nmid \mid$ Out $(H) \mid$, a contradiction. Hence $r \neq p$.

Let $\exp _{r}(q)=t$, then $t \mid r-1$. By [7, P.190] and [8, P.502] we have

$$
(*)\left(q^{n}-1\right)_{r}=\left\{\begin{array}{cc}
\left(q^{t}-1\right)_{r}\left(\frac{n}{t}\right)_{r}, & \text { if } t \text { divides } n ; \\
1, & \text { if } t \text { does not divide } n .
\end{array}\right.
$$

It is well known that if $(b, r-1)=1$, then $r \mid q^{b d}-1$ if and only if $r \mid q^{d}-1$. Hence

$$
\text { (**) }\left(q^{n r^{s}}-1\right)_{r}=\left\{\begin{array}{cc}
\left(q^{n}-1\right)_{r} r^{s}, & \text { if } r \text { divides } q^{n}-1 ; \\
1, & \text { if } r \text { does not divide } q^{n}-1 .
\end{array}\right.
$$

Assume that $H={ }^{2} A_{2}(q)$. If $r \mid d g$, then $r=3$ and $r \mid q+1$. We have $q \equiv-1(\bmod r)$ and so $3 \mid q^{2}-q+1$. Thus

$$
\left|H_{3}\right|=\frac{1}{3}\left(q^{2}-1\right)_{3}\left(q^{3}+1\right)_{3}=\frac{1}{3}(q+1)_{3}^{2}\left(q^{2}-q+1\right)_{3} \geq 3^{2},
$$

a contradiction. Assume that $r \nmid d g$. Then $r \mid f$. Let $f=r^{s} k$ with $(k, r)=1$. Assume that $r \mid q+1$. By previous argument, we may assume that $r>3$. Thus

$$
\left|H_{r}\right|=\left(q^{2}-1\right)_{r}\left(q^{3}+1\right)_{r}=(q+1)_{r}^{2}\left(q^{2}-q+1\right)_{r} \geq r^{2},
$$

a contradiction. Hence we may assume that $r \nmid q+1$ and so $t \in\{1,6\}$.

When $t=1$,

$$
\left|H_{r}\right|=\left(q^{2}-1\right)_{r}\left(q^{3}+1\right)_{r} \geq\left(p^{f}-1\right)_{r}=\left(p^{k}-1\right)_{r} r^{s} \geq r^{s+1},
$$

a contradiction.

When $t=6,\left(q^{3}-1\right)_{r}=1$. By $(* *)$,

$$
\left|H_{r}\right|=\left(q^{3}+1\right)_{r}=\left(p^{6 f}-1\right)_{r}=r^{s}\left(p^{6 k}-1\right)_{r} \geq r^{s+1},
$$

again a contradiction.

Assume that $H=D_{4}(q)$. Suppose that $r \mid g d$. Since $r>2$, we have $r=3$. Since $3 \mid q^{2}-1$, by $\left|D_{4}(q)\right|=\frac{1}{(2, q-1)^{2}} q^{6}\left(q^{4}-1\right)^{2}\left(q^{2}-1\right)$, we have $r^{3}|| H \mid$, a contradiction. Hence we may assume that $r \nmid g d$ and $r \mid f$. By (*), it is easy to obtain that $\left|H_{r}\right|>r$, a contradiction.

From now, we assume that $H \notin\left\{{ }^{2} A_{2}(q), D_{4}(q)\right\}$. 
Suppose that $r \mid d g$. Since $r>2$ and $g \in\{1,2\}$, we have $r \mid d$ and $H$ is one of simple groups $A_{n}(q)(n>1),{ }^{2} A_{n}(q), E_{6}(q)$ with $r=3,{ }^{2} E_{6}(q)$ with $r=3$. If $H=E_{6}(q)$, then $r \mid q-1$; if $H={ }^{2} E_{6}(q)$, then $r \mid q+1$; if $H=A_{n}(q)$, then $r \mid q-1$ and $n \geq 2$; if $H={ }^{2} A_{n}(q)$, then $r \mid q+1$ and $n \geq 4$, it is easy to obtain that $r^{2}|| H \mid$ from (*), a contradiction.

Suppose that $r \nmid d g$, then $f=r^{s} k$ with $s \geq 1$ and $(k, r)=1$. Let $\exp _{r}(q)=c$. From the orders of Lie type simple groups, we have $q^{c}-1|| H \mid$ if $c$ is odd or $q^{\frac{1}{2} c}+1|| H \mid$ if $c$ is even.

When $c$ is odd, by (**)

$$
\left|H_{r}\right| \geq\left(q^{c}-1\right)_{r}=\left(p^{k r^{s} c}-1\right)_{r}=\left(p^{k c}-1\right)_{r} r^{s} \geq r^{s+1},
$$

a contradiction.

When $c$ is even, by $r \nmid q^{\frac{1}{2} c}-1$ and (**), we have

$$
\left|H_{r}\right| \geq\left(q^{\frac{1}{2} c}+1\right)_{r}=\left(q^{c}-1\right)_{r}=\left(p^{k r^{s} c}-1\right)_{r}=\left(p^{k c}-1\right)_{r} r^{s} \geq r^{s+1},
$$

a final contradiction.

This completes the proof of the lemma.

\section{The proof of main result}

Suppose that the theorem is false and $G$ is a counter-example with minimal order. We will derive a contradiction in several steps.

STEP 1. $O_{p^{\prime}}(G)=1$.

Denote $N=O_{p^{\prime}}(G)$. If $N>1$, we consider the factor group $G / N$. Obviously, $P N / N$ is a Sylow $p$-subgroup of $G / N$, which is isomorphic to $P$, so $P N / N$ has the same smallest generator number as $P$, i.e., $d_{p}$ and so

$$
\mathcal{M}_{d_{p}}(P / N)=\left\{P_{1} / N, \ldots, P_{d_{p}} / N\right\}
$$

We know that every $P_{i} / N$ is also a CAP-subgroup of $G / N$ by Lemma 2.1. Thus $G / N$ satisfies the hypotheses of the theorem. We have that either $P N / N$ is of order $p$ or $G / O_{p^{\prime}}(G)$ is $p$-supersolvable by the choice of $G$, it follows that either $P$ is of order $p$ or $G$ is $p$-supersolvable, a contradiction. Thus, we have $N=O_{p^{\prime}}(G)=1$, as desired. 
STEP 2. $P$ is non-cyclic.

If $P$ is cyclic, then the unique maximal subgroup $\Phi(P)$ of $P$ is CAPsubgroup in $G$ by the hypotheses. Hence either $P$ is of order $p$ or $G$ is $p$ supersolvable by [1, Theorem 3.2], a contradiction.

STEP 3. $\Phi(P)_{G}=1$, therefore, $O_{p}(G)$ is an elementary abelian group.

If not, take any $T \leq \Phi(P)_{G}$ such that $T \unlhd G$. We consider the factor group $G / T$. Since every maximal subgroup of $P$ contains $\Phi(P)$ and $P / T$ has the same smallest generator number as $P$, so

$$
\mathcal{M}_{d_{p}}(P / T)=\left\{P_{1} / T, \ldots, P_{d_{p}} / T\right\}
$$

We know that every $P_{i} / T$ is also a CAP-subgroup of $G / N$ by Lemma 2.1. Thus, $G / T$ satisfies the hypotheses of the theorem. Hence, either $P / T$ is of order $p$ or $G / T$ is $p$-supersolvable by the choice of $G$. If $P / T$ is of order $p$, then $P$ is cyclic, contrary to Step 2. Hence $G / T$ is $p$-supersolvable, then $G$ is $p$-supersolvable, a contradiction.

STEP 4. If $N$ is minimal normal in $G$ contained in $P$, then $|N|=p$.

By Lemma 2.3(2).

STEP 5. All minimal normal subgroups of $G$ are contained in $O_{p}(G)$.

Assume that $H$ is a minimal normal subgroup of $G$ which is not a $p$ subgroup. As $O_{p^{\prime}}(G)=1$ by Step 1, we have that $p \| H \mid$ and $H$ is non-abelian characteristic simple group. Then

(5.1) All $P_{i} \in \mathcal{M}_{d_{p}}(P)$ avoid the chief factor $H / 1, H$ is a non-abelian simple group with $\left|H_{p}\right|=p$.

By Lemma 2.3(1) we know that $\left|H_{p}\right|=p$. So $H$ is a non-abelian simple group. Obviously $H$ is avoided by every $P_{i} \in \mathcal{M}_{d_{p}}(P)$.

(5.2) $O_{p}(G)=1$.

If $O_{p}(G) \neq 1$, we can pick a minimal normal subgroup $N$ of $G$ contained in $O_{p}(G)$. By Step 4 we know that $N$ is of order $p$. Consider the chief series of $G$ :

$$
1 \triangleleft N \triangleleft N H \triangleleft \cdots \triangleleft G \text {. }
$$

For an arbitrary $P_{i} \in \mathcal{M}_{d_{p}}(P)$, since $P_{i}$ avoids $H N / N, P_{i}$ must cover $N$ by 
Lemma 2.1(3). Hence $N \leq P_{i}$. Then

$$
N \leq \bigcap_{i=i}^{d_{p}} P_{i}=\Phi(P),
$$

which is contrary to Step 3.

(5.3) $C_{G}(H)=1$.

Suppose that $C_{G}(H) \neq 1$. Now we pick a minimal normal subgroup $H^{*}$ of $G$ contained in $C_{G}(H)$. Then $H \cap H^{*}=1$. For any $P_{i} \in \mathcal{M}_{d_{p}}(P)$, we know that $P_{i}$ avoids $H, P_{i}$ must cover $H^{*}$ by Lemma 2.1(3). Therefore, $H^{*}$ is a group of order $p$, which is contrary to (5.2).

(5.4) $G=P H$.

By (5.3), we know that the non-abelian simple group $H$ is the unique minimal normal subgroup of $P H$. So all chief factors of $P H$ are $H / 1$ or a cyclic group of order $p$. By (5.1), we know that all $P_{i} \in \mathcal{M}_{d_{p}}(P)$ cover or avoid all chief factors of $P H$. So $P H$ satisfies the hypothesis of the theorem. If $P H<G$, then either $P$ is of order $p$ or $P H$ is $p$-supersolvable by the minimal choice of $G$. If $P H$ is $p$ supersolvable, then $H$ is $p$-supersolvable. But this is contrary to (5.1). Hence $G=P H$.

(5.5) Finishing the proof of (5).

By (5.3) we have $C_{G}(H)=1$. Then $G$ and $G / H$ are isomorphic to a subgroup of $A u t(H)$ and a subgroup of $A u t(H) / \operatorname{Inn}(H)$, respectively. This means that $H_{p}$ is of order $p$ and $p$ divides the order of $O u t(H)$. By Lemma 2.4 , this is impossible.

STEP 6. $G=O_{p}(G) \times M$, the semi-direct product of $O_{p}(G)$ with a subgroup $M$ of $G$ and $O_{p}(G)$ is a direct product of normal subgroups of $G$ of order $p$.

By Lemma 2.3(3).

STEP 7. The final contradiction.

Since $N \leq Z(P)$ for any minimal normal subgroup $N$ of $G, P \leq C_{G}\left(O_{p}(G)\right.$ ). Since $C_{G}\left(O_{p}(G)\right) \cap M \triangleleft\left\langle O_{p}(G), M\right\rangle=G, C_{G}\left(O_{p}(G)\right) \cap M=1$ by Step 4 and 5 . Then $P \cap M=1$. This implies that $P=P \cap O_{p}(G) M=O_{p}(G)(P \cap M)=$ $O_{p}(G)$. Therefore by Step 6 we have that $G$ is $p$-supersolvable, the final contradiction. 
REMARK. The authors do not know the proof without using the classification of finite simple groups.

\section{Applications}

We give some applications of our main result.

Suppose that $p$ is the smallest prime dividing the order of $G$. We know that $G$ is $p$-nilpotent if $G_{p}$ is cyclic by [7, IV Satz 2.8] and $p$-supersolubility implies the $p$-nilpotency. By our main result we immediately have the following corollary.

Corollary 4.1. Let $p$ be the smallest prime dividing $|G|$ and $P$ a Sylow p-subgroup of $G$. Then $G$ is p-nilpotent if and only if every member in $\mathcal{M}_{d_{p}}(P)$ is a CAP-subgroup of $G$.

CoRollary 4.2. Suppose that $P$ is a Sylow p-subgroup of $G$ and $N_{G}(P)$ is p-nilpotent for some prime $p \in \pi(G)$. Then $G$ is p-nilpotent if and only if every member in $\mathcal{M}_{d_{p}}(P)$ is a CAP-subgroup of $G$.

Proof. We only need to prove the "if" part.

By our main result we know that either $P$ is cyclic or $G$ is $p$-supersolvable. If $P$ is cyclic, then we have $N_{G}(P)=C_{G}(P)$. Applying Burnside's $p$-nilpotence criterion ([7, Hauptsatz IV.2.6]), we get that $G$ is $p$ nilpotent. Now suppose that $G$ is $p$-supersolvable. Since the $p$-length of $p$-supersolvable groups is at most 1 , we have $P O_{p^{\prime}}(G)$ is normal in $G$. Set $\bar{G}=G / O_{p^{\prime}}(G)$. Then $\bar{G}=N_{\bar{G}}(\bar{P})=N_{G}(P) O_{p^{\prime}}(G) / O_{p^{\prime}}(G)$ is $p$-nilpotent by hypothesis. Hence $G$ is $p$-nilpotent, as desired.

Suppose that $G$ is $p$-solvable. If Sylow $p$-subgroups of $G$ are cyclic, then $G$ is $p$-supersolvable. Therefore, immediately from our main result, we have the following corollary which is a generalization of $[3$, Theorem A].

Corollary 4.3. Suppose that $G$ is a p-solvable group, where $p$ is a fixed prime number in $\pi(G)$, and $P$ is a Sylow p-subgroup of $G$. Then $G$ is p-supersolvable if every member in $\mathcal{M}_{d_{p}}(P)$ is a CAPsubgroup of $G$.

The following is a generalization of [3, Theorem C]. 
THEOREM 4.4. Suppose that $G$ is a group. Then $G$ is supersolvable if and only if every member in $\mathcal{M}_{d_{p}}(P)$ is a CAP-subgroup of $G$ for every prime $p$ in $\pi(G)$ and for every Sylow p-subgroup $P$ of $G$

PROOF. We only need to prove the "if" part.

By Corollary 4.3 it is sufficient to prove that $G$ is solvable. Hence we want to prove that every chief factor of $G$ is solvable. Suppose that $L / K$ is an arbitrary chief factor of $G$. For any prime $p \in \pi(L / K)$, we know that there exists a maximal subgroup $H$ of a Sylow $p$-subgroup of $G$ such that $H$ either covers or avoids $L / K$. If $H$ covers $L / K$, obviously $L / K$ is solvable. Hence assume that $H$ avoids $L / K$. This implies that $|L / K|_{p} \leq p$. Therefore, every Sylow subgroup of $L / K$ is of prime order. Hence $L / K$ is solvable.

This completes the proof of Theorem 4.4.

Acknowledgments. Part of this research was carried out during a visit of the third author to Departamento of Matemáticas, Universidad Pública de Navarra, Pamplona, Spain, in March, 2010. He is grateful to the Department for its warm hospitality.

\section{REFERENCES}

[1] A. Ballester-Bolinches - L. M. Ezquerro - A. N. Skiba, Local embeddings of some families of subgroups of finite group, Acta Math. Sinica, Eng. Ser., 25: pp. 869-882, 2009.

[2] J. H. Conway - R. T. Curtis - S.P. Norton - R. A. Parker - R. A. Wilson, ATLAS of Finite Groups, Clarendon Press, Oxford, 1985.

[3] L. M. EzQUERro, A contribution to the theory of finite supersolvable group, Rend. Sem. Mat. Univ. Padova, 89: pp. 161-170, 1993.

[4] W. Gaschütz, Praefrattini Gruppen, Arch. Math. 13: pp. 418-426, 1962.

[5] J. K. GILlam, Cover-avoid subgroups in finite groups, J. Algebra, 29: pp. 324$329,1974$.

[6] Guo Xiuyun - K. P. Shum, Cover-avoidance properties and the structure of finite groups, J. Pure Applied Algebra, 181: pp. 297-308, 2003.

[7] B. Huppert, Endliche Gruppen I. Berlin: Springer-Verlag, 1968.

[8] B. Huppert - N. Blackburn, Endiche Gruppen II, Spring-Verlag, Berlin, 1979.

[9] Y. LI, On cover-avoiding subgroups of Sylow subgroups of finite groups, Rend. Sem. Mat. Univ. Padova, 123: pp. 249-258, 2010.

[10] S. LI - X. He, On Normally Embedded Subgroups of Prime Power Order in Finite Groups, Comm. Algebra, 36: pp. 2333-2349, 2008. 
[11] Y. LI - G. WANG, A note on maximal CAP-subgroups of finite groups, Algebras, Groups and Geometries, 23: pp. 285-290, 2006.

[12] J. Petrillo, The cover-avoidance property in finite groups, Ph.D. thesis, State University of New York at Binghamton, 2003.

[13] J. Petrillo, CAP-subgroups in a direct product of finite groups, J Algebra, 306: pp. 432-438, 2006.

[14] K.U. Schaller, Über Deck-Meide-Untergruppen in endlichen auflösbaren Gruppen, Ph.D. thesis, University of Kiel, 1971.

Manoscritto pervenuto in redazione il 3 Agosto 2012. 
